\title{
COMPARISON OF IMMUNOFLUORESCENCE ASSAYS RESULTS FOR DIAGNOSIS OF RICKETTSIAL DISEASES USING DIRECT SERUM AND FILTER PAPER SERUM SPOTS
}

\author{
SWEETY AA ${ }^{1}$, MUNSHI SU ${ }^{2}$, HOSSAIN MZ ${ }^{3}$, ISLAM B ${ }^{4}$, TABASSUM $\mathrm{S}^{5}$, AHMED I ${ }^{6}, \mathrm{KHAN} \mathrm{S}^{7}$, \\ KHAN AHMEA ${ }^{8}$, AZAD KAK ${ }^{9}$
}

\begin{abstract}
:
Background: Rickettsial diseases are difficult to diagnose in Bangladesh due to low index of suspicion and lack of diagnostic facilities in most laboratories especially in rural areas. Immunofluorescence assay (IFA) is the gold standard serological test for diagnosis of Rickettsial diseases. For serological diagnosis of rickettsial diseases, use of filter paper is an inexpensive and convenient method for collecting, storing, and transporting of blood samples with little space without refrigeration. Current study shows that the rickettsial diseases were present in febrile patients in Bangladesh. The study also compared the results of direct serum by IFA and filter paper serum spot followed by IFA as an alternative way of testing frozen samples.
\end{abstract}

Materials and Methods: This observational study for evaluation of rickettsial diseases from direct serum by IFA and sera dried on blotting paper followed by IFA at the department of Virology, Bangabandhu Sheikh Mujib Medical University (BSMMU), Dhaka, Bangladesh. A total of 152 patients' sera were selected by systemic random sampling of every $3^{\text {rd }}$ sera of 556 febrile patients who were tested negative for dengue and or Chikungunya virus infection. Forty cases were confirmed by IFA from direct sera. In addition, 60 samples were selected from 152 sera by systemic random sampling of every $3^{\text {rd }}$ sera for blotting on filter paper for further IFA test. Out of 60 sera, 35 were confirmed by IFA from direct sera and of the 35 confirmed positive cases, $31(88.6 \%)$ were positive by IFA after blotting paper assay. Taking the IFA of direct sera as gold standard test, the sensitivity, specificity, positive predictive value and negative predictive value of IFA from blotting paper eluted sera were 88\%, 100\%, 100\% and 86\% respectively.

Conclusion: The study showed the use of filter paper is an inexpensive and convenient method for storing and transporting samples for serological tests like IFA in diagnosis of rickettsial diseases.

Keywords: Rickettsial diseases, Immunofluorescence assay, Filter paper serum blot.

J Dhaka Med Coll. 2019; 28(2) : 153-158

\section{Introduction:}

Rickettsial diseases are re-emerging in Asiatic tropics ${ }^{1}$ and causing serious public health problems now a day. Evidence of rickettsial disease is found from Assam, West Bengal, Himachal Pradesh and other provinces of India, surrounding Bangladesh. ${ }^{2}$ There are few available published data regarding the presence of rickettsial diseases from Bangladesh are also found. $3,4,14,15$

Rickettsial diseases are the oldest known vectorborn diseases ${ }^{6}$ and may cause serious public health problems. Morbidity and mortality rates vary among various types of rickettsial diseases. ${ }^{6}$ In untreated cases, mortality rate of rickettsial diseases may be as high as $30-35 \% .{ }^{7}$

1. Dr. Afroza Akbar Sweety, Assistant Professor, Department of Virology, Dhaka Medical College, Dhaka, Bangladesh;

2. Prof. Dr. Saif Ullah Munshi, Professor and Chairman, Department of Virology, Bangabandhu Sheikh Mujib Medical University, Dhaka, Bangladesh.

3. Dr. Mohammad Zaid Hossain, Associate Professor of Medicine, Dhaka Medical College.

4. Dr. Baishakhi Islam, Associate Professor of Pharmacology \& Therapeutics, Gazi Medical College, Khulna.

5. Prof. Dr. Shahina Tabassum, Professor, Department of Virology, Bangabandhu Sheikh Mujib Medical University, Dhaka, Bangladesh.

6. Dr. Imtiaz Ahmed, Indoor Medical Officer, Sir Salimullah Medical College, Dhaka

7. Dr. Shahjalal Khan, Consultant Internal Medicine, Momtaj Trauma Centre, Mohammadpur, Dhaka

8. Dr. AHM Ejrarul Alam Khan, Assistant Registrar Medicine, Dhaka Medical College, Dhaka

9. Prof. Khan Abul Kalam Azad, Professor of Medicine \& Principal, Dhaka Medical College, Dhaka

Correspondence: Dr. Afroza Akbar Sweety, Assistant Professor, Department of Virology, Dhaka Medical College, Dhaka, Bangladesh, Cell phone: 01742430042, Email: afrozasweety98@yahoo.com 
Clinical features of Rickettsial diseases are nonspecific and resemble many febrile illnesses e.g. dengue, chikungunya, measles, rubella, typhoid, malaria etc. The common symptoms of rickettsial diseases are fever, malaise, headache, myalgia and rash. ${ }^{8}$ Though clinical symptoms are similar among these diseases; pathogenesis and treatment of rickettsial diseases are different. Thus, a vast number of febrile cases remain undiagnosed. The objective for this study was to check whether there was a silent outbreak of rickettsial diseases in Dhaka and blotting paper serum blot assay was used as an alternate of transport of frozen samples.

Bangladesh is a resource-poor developing country where epidemiology and burden of rickettsial disease is largely undiscovered. Tests like immunofluorescence assay the "gold standard" technique for diagnosis of rickettsial diseases is used as the reference technique in most laboratories. ${ }^{9}$ This test is both sensitive and specific which detects IgM, IgG, and IgA specific antibodies to rickettsial antigens. This test needs advanced laboratory facilities ${ }^{10}$ as well as skilled personnel which are available only at tertiary level hospitals.

Filter paper blood spot-based assays have been developed for diagnosis of Rickettsial disease. ${ }^{11}$ Peripheral rural areas have insufficient access to diagnostic laboratory facilities, amenities and difficulty in transporting frozen specimen. ${ }^{12}$ For serological diagnosis in developing countries like Bangladesh, many difficulties have to be faced in rural areas e.g. refrigeration of samples in proper temperature due to interruption in electricity in most of the laboratories. Under these circumstances, blood sample collected by finger or heel pricking and blotted on filter paper may be a convenient and cheap alternative of sending frozen diagnostic samples for IFA to the Central tertiary level hospitals.

Rickettsial diseases are treatable, when diagnosed properly. However, reference laboratories play a vital role by testing the samples dried on filter paper sent from peripheral rural areas. For this purpose, we applied IFA technique adapted to dried sera collected on blotting paper. The purpose of this study was to confirm the presence of rickettsial diseases from direct serum sample by IFA and sera collected on blotting paper and eluted sera tested by IFA.

\section{Methods:}

This observational study of serological diagnosis of rickettsial diseases was conducted at the department of Virology, BSMMU, from January to December, 2012. The study protocol was approved by Institutional Review Board (IRB) of BSMMU. Total 985 patients with recent febrile illness were included. Five milliliters of whole blood for serum was collected from patients. Sera were tested by Immune Chromatographic Test (ICT) for IgM of Dengue and or Chikungunya and 556 sera which were ICT negative were selected. A total of 152 sera were selected for IFA from 556 sera by systemic random sampling of every $3^{\text {rd }}$ sample $(\mathrm{N}=556$, $\mathrm{n}=152 \mathrm{~K}=3)$. They were kept in $-20^{\circ} \mathrm{C}$ until further use. For sera blotted on filter paper followed by IFA test, 60 samples were selected from these 152 samples by systemic random sampling of every $3^{\text {rd }}$ sera and of them, 35 were confirmed positive by IFA in direct serum.

\section{Immunofluorescence tests:}

For direct serum IFA, positive control serum was diluted to $1: 100$ in $2 \%$ Bovine serum albumin (BSA)-phosphate buffered saline (PBS) and added in first column of wells and negative control serum was diluted to $1: 128$ in $2 \%$ BSAPBS to the last column. Dilution was done prior to use. Then $2 \mu \mathrm{l}$ of test sera (all in duplicate) which were diluted to $1: 128$ in $2 \%$ BSA, were putting on each slide to cover the well. The slide was incubated for 30 minutes at $37^{\circ} \mathrm{C}$ in a humidified container and washed 3 times with PBS for 5 minutes. After washing, slides were dried, $2 \mu$ l of conjugate was added, before adding to each well conjugate need to be diluted to $1: 100$ in $2 \%$ BSA-PBS. Slides were again incubated for 30 minutes at $37^{\circ} \mathrm{C}$ in a humidified container, washed 3 times with PBS for 5 minutes and dried. Mounting fluid and large cover slip was added to cover all wells. We conducted IFA by using dedicated IFA slides, positive control, negative control and conjugate from the Australian Rickettsial Reference Laboratory.

\section{Blotting-paper test:}

Filter paper serum spot based assay was performed by adding $25 \mu$ of serum dropped onto filter paper covering a circle of about $13 \mathrm{~mm}$ 
ProteinsaverTM (Whatman, Maidstone, United Kingdom). Then filter strips were left to air dry and kept at room temperature for 72 hours. A cardpunch (Kangaro No 376224, India) was used to cut $6 \mathrm{~mm}$ diameter discs from the seraimpregnated filter paper sera spots, halfway between the center and the edge of the blood spot the strip and serum was eluted with 250 $\mu l$ of $\mathrm{PBS}$ at $37^{\circ} \mathrm{C}$ for overnight. Then, the eluted serum was subjected to IFA and IFA was done following the previously described procedure.

\section{Interpretation of results of IFA:}

The cut-off of IFA positive result was 1:128 (Australian Rickettsial Reference Laboratory Foundation Ltd., Geelong, Victoria, Australia). Rickettsial IF was read by checking positive control wells first. All rickettsial antigens from the three groups were fluoresced. The negative control wells were checked after the positive control. Slide was read with an epifluorescent microscope (Olympus EX50, Japan) at 400x magnification.

\section{Pictures with Immunofluorescence Microscope (Olympus-EX50):}

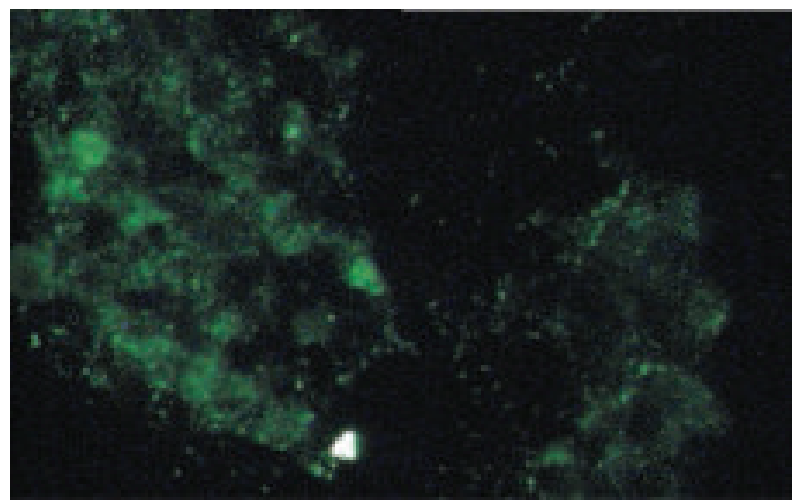

Fig.-1: Positive control in Immunofluorescence Microscope (Olympus-EX50)

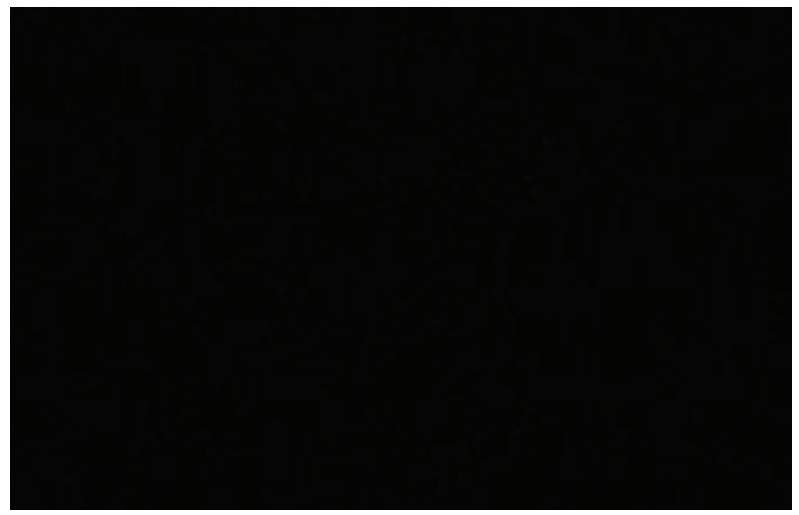

Fig.-2: Negative control in Immunofluorescence Microscope (Olympus-EX50)

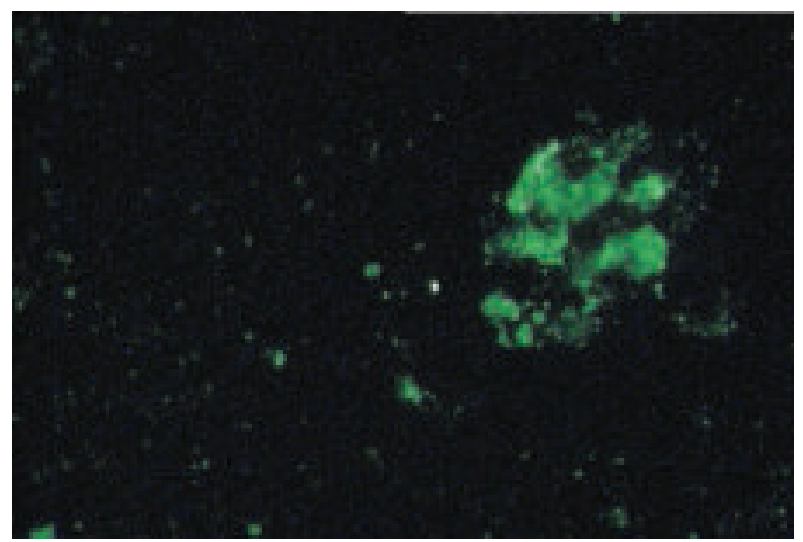

Fig.-3: Positive result for rickettsial antibody from direct serum

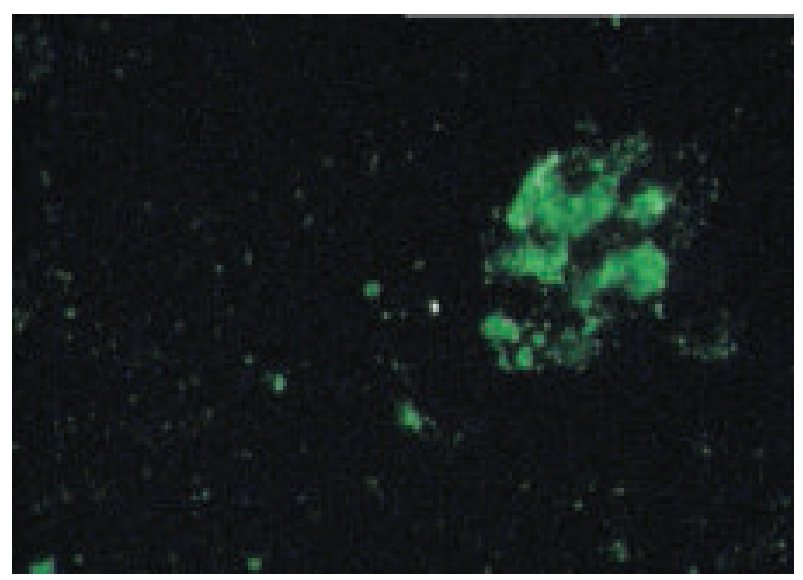

Fig.-4: Positive result for rickettsial antibody after blotting of serum in filter paper

\section{Data analysis:}

Data were interpreted and analyzed by using Microsoft EXCEL, (2007, USA) and SPSS version 17.0. Inter-rater reliability analysis was done by kappa test; kappa value was 0.86 at $95 \%$ CI.

\section{Results:}

Characteristics of study population and results of IFA are shown in Table-I. The age ranges of patients were from 17 to 57 years with mean age of $28.18 \pm 10.16( \pm \mathrm{SD})$ years. The number of male was 89 (58.6\%) and female was 63 (41.4\%). Among the study population, 40 $(26.3 \%)$ were positive by gold standard serological test (IFA), were reactive at a cut off titer of $1: 128$. 
Table- I

Characteristics of study population and tests performed.

\begin{tabular}{lc}
\hline Variables & Results \\
\hline Total number of febrile patients & 556 \\
Total number of study subjects & 152 \\
for IFA & \\
Age (Mean \pm SD) & $28.12 \pm 10.16$ \\
Gender (male : female) & $1.41: 1$ \\
IFA Positive & $40(26.3 \%)$ \\
\hline
\end{tabular}

IFA positive results among different bio-groups of rickettsial diseases are shown in table II. typhus group (TG) were 14 (35\%), scrub typhus group (STG) were 13 (32.5\%), spotted fever group (SFG) were $10(25 \%)$ and $3(7.5 \%)$ samples were positive in intermediate (spotted fever group + typhus group) group out of 40( 100\%) positive patients.

Table-II

IFA positive (1:128) results of Rickettsial disease in different bio-groups $(n=40)$

\begin{tabular}{lc}
\hline Bio-groups & No of sample \\
\hline SFG positive & $10(25 \%)$ \\
TG positive & $14(35 \%)$ \\
STG positive & $13(32.5 \%)$ \\
*(SFG+TG) positive & $3(7.5 \%)$ \\
Total IFA positive & $40(100 \%)$ \\
\hline
\end{tabular}

*(SFG+TG) positive means 3 sera showed reaction both in SFG and TG

These 3 were deducted from the total $(43-3=40)$

Taking the direct serum IFA as the gold standard test, the sensitivity and specificity of blotting paper sera assay following IFA were $88 \%$ and $100 \%$, respectively, with a positive predictive value of $100 \%$, and a negative predictive value of $86 \%$ shown in table-III. There were no statistically significant differences between the IFA of direct sera and IFA after blotting of sera on filter paper. In Inter-rater reliability analysis by kappa test, Kappa value was 0.86 done by SPSS version 17.0 at $95 \%$ CI.

\section{Table- III}

Sensitivity, Specificity, PPV and NPV of IFA after blotting paper serum spots, Kappa statistics (95\% CI) for inter-rater agreements when IFA was gold standard test. IFA after serum blotted on blotting paper and IFA from direct sera

\begin{tabular}{lccc}
\hline $\begin{array}{l}\text { IFA after serum } \\
\text { blotted on blotting }\end{array}$ & \multicolumn{2}{c}{ IFA from direct sera } & Total \\
paper & & & \\
\hline Positive & Negative & \\
Negative & 41 & 0 & 31 \\
Total & 35 & 25 & 29 \\
Sensitivity - & & $88 \%$ & 60 \\
Specificity & & $100 \%$ & \\
$\quad$ * PPV & & $100 \%$ & \\
$* *$ NPV & & $86 \%$ & \\
$\quad$ Kappa Value & & 0.86 & \\
\hline
\end{tabular}

${ }^{*} \mathrm{PPV}=$ Positive predictive value $* * \mathrm{NPV}=$ Negative predictive value

\section{Discussion:}

Rickettsial diseases are a growing concern in public health and exist worldwide in endemic foci. They are also prevalent in neighboring countries like India and Myanmar, and as such, transmissions of rickettsial diseases are also suspected in Bangladesh. Rickettsial diseases are reemerging, and factors influencing this reemergence include the immuno depression associated with declines in social conditions brought about by factors such as poor hygiene, poverty, or war. ${ }^{13}$

In the present study, Rickettsial antibodies were detected by IFA among the suspected febrile patients. In Bangladesh, rickettsial diseases were reported among the febrile hospitalized patient's sera at Mymensingh Medical College Hospital by using WF test. ${ }^{5}$ Presence of rickettsial diseases in Chittagong was detected ${ }^{14}$. The presence of rickettsial pathogens was investigated in eight places in all the divisions of Bangladesh. ${ }^{15}$ 
In the present study, $(26.1 \%)$ cases were confirmed among three bio-groups of rickettsial diseases by IFA from direct sera in febrile ill patients. The most frequent bio-group (35\%) was typhus group followed by (32.5\%) were scrub typhus group and $(25 \%)$ were spotted fever group and $(7.5 \%)$ were intermediate group (TG/ SFG) of rickettsioses. Similar study from Vientiane and Laos showed most frequency in typhus group (43\%) followed by scrub typhus group (28\%). ${ }^{13}$ Studies from Thai-Myanmar border, Sri Lanka, Sweden; Peru had shown dissimilarity in frequency among the bio-groups of rickettsiae. ${ }^{5,16-18}$ In a study from Korea showed $(36.9 \%)$ of cases were scrub typhus group, $(46.7 \%)$ were either spotted fever group or murine typhus group and (16.3\%) were indeterminate ${ }^{19}$. In a study among international travelers in Oslo, Norway showed (82.5\%) had spotted fever group, (5.7\%) had scrub typhus group, (3.6\%) had typhus group, (1.4\%) had indeterminate typhus group / spotted fever group rickettsioses. ${ }^{20}$ Disparity in prevalence rate among the bio-groups of rickettsial diseases may be due to the presence of different vectors and reservoirs in different geographical areas that transmit rickettsial organism to human. In the present study, the frequency of typhus group was slightly higher than others but was not statistically significant. In the present study, samples were not distributed regionally and vectors of rickettsial diseases were not studied. Thus, the bio-group data may not represent the real scenario of the whole country. However, the present study showing data among biogroups of rickettsial diseases in Bangladesh.

The filter papers are light, cannot be broken or split, can be stored at room temperature for several weeks, require small storage space, and can be shipped by mail. The serum spot technique can be performed anywhere including rural areas by minimally trained personnel and therefore is suited to screening programs in developing regions such as Bangladesh. Though a very little is known about the recovery of rickettsial antibodies from serum samples dried on filter paper.
In the present study, the sensitivity of IFA after blotting paper serum spots was $88 \%$, specificity was $100 \%$, PPV was $100 \%$, and NPV was $86 \%$. There was no statistically significant difference between the results of patients' sera having rickettsial diseases, in serum samples and serum on filter paper at room temperature by IFA ( $\mathrm{P}>0.5$, Kappa test). In a similar study from India for murine typhus sensitivity of $91 \%$, specificity of $100 \%$, PPV of $100 \%$ and a NPV of $80 \%$ were observed by IFA using filter paper blood samples in comparison to serum samples. SFG patients had sensitivity of $95 \%$, specificity of $88 \%$, PPV of $95 \%$ and a NPV of $87 \%$. There were no statistically significant differences observed among patients by the two techniques. ${ }^{21}$ Another study from Philippines also showed the similar results with the present study. ${ }^{5}$ Overall, the result of this study suggests that sera collection on filter paper can be effectively substituted for direct serum sampling in rickettsial-antibody assays. This method of specimen collection provides an economical way to obtain and transport specimens without decreasing the sensitivity or specificity. ${ }^{22}$ Moreover, testing blood or serum blotted on filter paper may be a challenging first step to explore and confirm outbreaks of rickettsial diseases all over the world.

Storage of sera on filter paper is a frequently used technique after blood collection in human serologic surveys. Serologic surveys are recently being used to study microbial ecology and study of epidemic and endemic diseases, especially in areas like Bangladesh where modern laboratory facilities are not available in rural area.

\section{Conclusion:}

The results of the present study showed the use of filter paper is an inexpensive and convenient method for storing and transporting samples for serological tests. The study also showed that rickettsial infections are present among the febrile patients in Bangladesh by a gold standard serological test. Thus, the active surveillance of rickettsial diseases is required to know the exact magnitude and distribution of vectors and diseases. There has been great progress in molecular biology, cellular biology 
and immunology of Rickettsia. However, diagnoses of rickettsial diseases still remain difficult. Rapid diagnostics methods are required for diagnosis of rickettsial diseases to prevent morbidity and mortality among Bangladeshi people.

\section{Limitation:}

Our study had some limitations. This was a hospital-based study, and therefore excluded acute febrile patients who never visited these hospitals. However, the selected study site was the regional referral hospital and had a wide catchment area.

\section{References:}

1. Rathi N \& Rathi A. Rickettsial infections: Indian perspective. Indian pediatrics. 2010;47(2):157-164.

2. Mittal V, Gupta N, Bhattacharya D, Kumar K, Ichhpujani RL, Singh S, et al. Serological evidence of rickettsial infections in Delhi. The Indian journal of medical research. 2012;135(4):538-541.

3. Faruque LI, et al. Prevalence and clinical presentation of Rickettsia, Coxiella, Leptospira Bartonella and chikungunya virus infections among hospital-based febrile patients from December 2008 to November 2009 in Bangladesh. BMC infectious diseases. 2017;17(1): 141 .

4. Miah MT, et al. Study on 40 cases of rickettsia. Mymensingh medical journal : MMJ. 2007;16(1):8588.

5. Parola P, et al. Warmer weather linked to tick attack and emergence of severe rickettsioses. PLoS neglected tropical diseases. 2008;2(11):e338.

6. Kelly DJ, et al. The past and present threat of rickettsial diseases to military medicine and international public health. Clinical infectious diseases : an official publication of the Infectious Diseases Society of America. 2002;34(Suppl 4):S145169.

7. Batra HV. Spotted fevers \& typhus fever in Tamil Nadu. The Indian journal of medical research. 2007;126(2):101-103.

8. Sexton DJ, et al. Rocky Mountain "spotless" and "almost spotless" fever: a wolf in sheep's clothing. Clinical infectious diseases : an official publication of the Infectious Diseases Society of America. 1992;15(3):439-448.

9. Blackwell LS, et al. Implicit theories of intelligence predict achievement across an adolescent transition: a longitudinal study and an intervention. Child development. 2007;78(1):246-263.
10. Mahajan SK et al., Relevance of Weil-Felix Test in Diagnosis of Scrub Typhus in India, JAPI vol 54 August 2006, 619-621.

11. Smit PW, et al. An overview of the clinical use of filter paper in the diagnosis of tropical diseases. The American journal of tropical medicine and hygiene. 2014;90(2):195-210.

12. Aung AK, et al., Rickettsial infections in Southeast Asia: implications for local populace and febrile returned travelers. The American journal of tropical medicine and hygiene. 2014;91(3):451-460.

13. Raoult D, et al. Rickettsioses as paradigms of new or emerging infectious diseases. Clinical microbiology reviews. 1997;10(4):694-719.

14. Kingston Hugh W, Hossain M, Leopold S,Anantatat T, Tanganuchitcharnchai A,Sinha I et al. Rickettsial Illnesses as Important Causes of Febrile Illness in Chittagong, Bangladesh. Emerging Infectious Diseases - www.cdc.gov/eid • 2018;Vol. 24, No. 4.

15. Chowdhury NF, Paul SK, Aung MS, Hossain MA, Ahmed F, Ahmed S., et al. Nationwide prevalence of Rickettsia felis infections in patients with febrile illness in Bangladesh. New Microbe and New Infect 2017; 19: 123-125.

16. Jensenius $M$, et al. Rickettsioses and the international traveler. Clinical infectious diseases : an official publication of the Infectious Diseases Society of America. 2004;39(10):1493-1499.

17. Schoeler GB, et al. Human spotted fever rickettsial infections. Emerging infectious diseases. 2005; $11(4): 622-624$.

18. Premaratna $\mathrm{R}$, et al. Clinically helpful rickettsial disease diagnostic IgG titers in relation to duration of illness in an endemic setting in Sri Lanka. BMC research notes. 2012;5:662.

19 Lee $\mathrm{N}$, et al. Risk factors associated with lifethreatening rickettsial infections. The American journal of tropical medicine and hygiene. 2008;78(6):973-978.

20. Jensenius M, et al. Threats to international travellers posed by tick-borne diseases. Travel medicine and infectious disease. 2006;4(1):4-13.

21 Phetsouvanh $\mathrm{R}$, et al. Comparison of indirect immunofluorescence assays for diagnosis of scrub typhus and murine typhus using venous blood and finger prick filter paper blood spots. The American journal of tropical medicine and hygiene. 2009;80(5):837-840.

22. Raoult D, et al. Antimicrobial therapy of rickettsial diseases. Antimicrobial agents and chemotherapy. 1991;35(12):2457-2462. 